\title{
VARIACIÓN GENÉTICA Y CONSERVACIÓN DE UNA POBLACION DE CROCODYLUS MORELETII EN CAUTIVERIO
}

\author{
Ricardo Serna-Lagunes ${ }^{1 *}$ \& Pablo Díaz-Rivera ${ }^{1}$ \\ ${ }^{1}$ Colegio de Postgraduados, Campus Veracruz. Apartado Postal 421. Km. 88.5 Carretera Federal \\ Xalapa-Veracruz, Predio Tepetates s/n, municipio de Manlio Fabio Altamirano. CP. 91700. Veracruz,

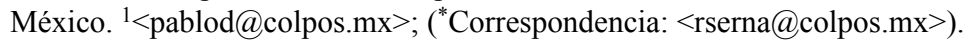

Serna-Lagunes, R. \& P. Díaz-Rivera. 2011. Variación genética y conservación de una población de Crocodylus moreletii en cautiverio. Acta Zoológica Mexicana (n. s.), 27(3): 547-563.

RESUMEN. La endogamia es un problema frecuente en poblaciones aisladas o en cautiverio, por esto es necesario implementar estrategias que minimicen su incidencia. El cocodrilo de pantano, Crocodylus moreletii, se aprovecha en cautiverio y la conservación de su variación genética es esencial para su explotación y en la toma de decisiones para la liberación de sus crías. El objetivo del estudio fue analizar la viabilidad poblacional, el riesgo de extinción y el tamaño efectivo de población de $C$. moreletii en cautiverio. Se utilizaron modelos de simulación y métodos directos y ecológicos con la finalidad de obtener las medidas demográficas y genéticas apropiadas para establecerse en los criaderos de C. moreletii. Se encontró que es factible establecer una población de 200 cocodrilos, lo que garantizará su sobrevivencia durante 50 años. El $N_{e}$ debe ser mayor a 900 cocodrilos, con una proporción de sexos de un macho por 2.5 hembras y con clase de talla adulto-reproductivo; con esto, se tendría un aproximado de $84 \%$ de cocodrilos reproductores efectivos en la población total, y presentarían una tasa de endogamia menor al 5.5\%. Esta información ayudará a conservar el $94 \%$ de la variación genética de la población de $C$. moreletii en cautiverio durante 100 años, y permitiría la transmisión de material genético viable a las crías para ser liberados al medio natural.

Palabras clave: Cocodrilo de Pantano, conservación ex situ, incremento de endogamia, viabilidad poblacional, tamaño efectivo de la población, liberación de cocodrilos.

Serna-Lagunes, R. \& P. Díaz-Rivera. 2011. Genetic variation and conservation of one population of Crocodylus moreletii in captivity. Acta Zoológica Mexicana (n. s.), 27(3): 547-563.

ABSTRACT. Inbreeding is a frequent problem in isolated or captive populations, therefore it is necessary to implement strategies to minimize its incidence. Morelet's crocodile, Crocodylus moreletii, is exploited in captivity and conservation of genetic variation is essential to its management and decision making in the release of offspring. The aim of this study was to analize population viability, extinction risk and effective population size of a $C$. moreletii captive population. We used simulation models and direct and ecological methods in order to obtain demographic and genetic measures appropriate for captive breeding practices of $C$. moreletii. It was found that it is feasible to establish a population of 200

Recibido: 03/07/2010; aceptado: 27/06/2011. 
crocodiles, which will ensure their survival for 50 years. $N_{e}$ must be greater than 900 , with a sex ratio of one male to 2.5 females and reproductive-adult size class. This would correspond to approximately $84 \%$ of effective reproductive crocodiles in the total population, having an inbreeding rate lower than $5.5 \%$. This information would help to conserve $94 \%$ of the population genetic variation of C. moreletii in captivity for 100 years, and would let the transmission of viable genetic material to their offspring to be released to the environment.

Key words: Morelet's crocodile, ex situ conservation, increase of inbreeding, population viability, effective population size, crocodile release.

\section{INTRODUCCIÓN}

La endogamia generalmente se manifiesta en poblaciones aisladas o en cautiverio por presentar un reducido tamaño efectivo de población $\left(N_{e}\right)$, y también es el resultado de cambios en la estructura y dinámica poblacional (Pray et al. 1994). Hartl \& Clark (1997) mencionan que si una población mantiene un reducido $N_{e}$, incluso por cortos periodos de tiempo, fundamentalmente causaría endogamia, teniendo como consecuencia una mayor proporción de individuos homocigotos. De tal forma se tendrían generaciones con menor variación genética por presentar copias de alelos que suelen ser idénticos por descendencia (Raybould et al. 2002). La endogamia impacta de manera negativa en las características de historia de vida como el crecimiento, la reproducción y la sobrevivencia, y en el potencial evolutivo, adaptativo y de especiación, a tal grado de llevar a las poblaciones a la extinción (Frankham 2000, Frankham et al. 2002). Ejemplos de especies con un reducido $N_{e}$ que trae consigo depresión genética por los efectos de la endogamia son los casos de Mirounga angustirostris (Hoelzel et al. 1993), Felis concolor (Hedrick 1995), Crocodylus niloticus (Flint et al. 2000) y Aquila adalberti (Ferrer et al. 2009), poblaciones que se encuentran en peligro de extinción.

Históricamente, las poblaciones de cocodrilianos fueron sometidas a una alta presión cinegética, y esto aunado a la fragmentación de su hábitat, condujo a disminuir sus tamaños poblacionales, inclusive algunas poblaciones estuvieron al borde de la extinción (Ross 1998). Ante la evidente problemática que experimentaron los cocodrilianos, algunos ejemplares se destinaron para ser conservados ex situ con la finalidad de recuperar sus poblaciones a posteriori (Huchzermeyer 2003). Al respecto, $\mathrm{Wu}$ et al. (2002), y Wang et al. (2006) indicaron que la prioridad en los sistemas de crianza de los cocodrilianos es conservar su variación genética de generación en generación; y es necesario que las crías seleccionadas para ser liberadas a su hábitat silvestre deben por lo menos tener una proporción de variación genética igual a la que se presenta en poblaciones naturales, para que puedan resistir y adaptarse a las presiones ambientales del hábitat en el que serán liberados (FitzSimmons et al. 2002).

En el contexto anterior, Crocodylus moreletii no es la excepción, ya que en México fue la especie que más sufrió los detrimentos poblacionales derivados de la caza ilegal para el comercio de su piel (Álvarez del Toro \& Sigler 2001). A nivel interna- 
cional, la especie se encuentra catalogada como amenazada por riesgo de extinción (IUCN 2010); en México, la legislación ambiental (NOM-059-SEMARNAT 2010) establece que está sujeta a protección especial y se debe conservar a través de dos estrategias: a) acatando el decreto de su veda y mediante prácticas de mejoramiento de su hábitat, y b) a través del rancheo para establecer núcleos reproductivos en los sistemas de producción en cautiverio denominados Unidad de Manejo para la Conservación de la Vida Silvestre (UMA), que también persigue el objetivo de recuperar aquellas poblaciones silvestres a través de la liberación de cocodrilos criados en estas unidades (INE 1997, SEMARNAT 1999). A la fecha, los programas de crianza de C. moreletii en las UMAs han arrojado resultados satisfactorios (Domínguez-Laso 2006); sin embargo, el objetivo de la liberación aun no se ha concretado debido a que se desconoce el nivel de variación genética de los individuos destinados para este fin, principalmente por el costo que implica el desarrollo de estudios con marcadores moleculares, tecnología que es la adecuada para examinar esta propiedad (Glenn 2002, Forstner \& Forstner 2002).

Para ello, otra manera para estimar directamente la variación genética es utilizando métodos directos y ecológicos-demográficos para determinar el $N_{e}$, el cual representa el número de ejemplares que se reproducen con éxito en una población y con el que se conserva la variación genética durante ciertos periodos de tiempo (Nunney \& Elam 1994). Es decir, el $N_{e}$ representa una medida que determina la tasa de disminución de la variación genética (Wright 1931), ya que se encuentra relacionada de manera inversa con el incremento en la tasa de endogamia $(\Delta F)$ (Caballero 1994); este concepto metodológico es el adecuado para aplicarse en las poblaciones destinadas a los sistemas de producción, ya que provee datos cuantitativos para generar los planes de manejo genético (Oldenbroek 1999). Otro concepto que está inmerso en la conservación de poblaciones es el tamaño poblacional mínimo viable (TMV), que representa un indicador del número mínimo de individuos que una población necesita para sobrevivir a lo largo del tiempo (Shaffer 1981). Este último concepto se ha aplicado ampliamente para la recuperación de poblaciones de especies en peligro de extinción (Rocha \& Gasca 2007). Debido a que el $N_{e}$ y el TMV se encuentran estrechamente relacionados (Frankham 1995, 1996), son parámetros considerados clave en la conservación de poblaciones (Ryman \& Laikre 1991, Hedrick \& Kalinowski 2000, Hedrick 2005), y su aplicación e implicaciones se reflejan en los estudios de aves (Payne 1990), mamíferos marinos (Marmontel et al. 2002), animales domésticos (Razmaité \& Sveistiené 2003), insectos (Schultz \& Hammond 2003) y peces (Zabel et al. 2006), arrojando resultados que propician su conservación y recuperación poblacional.

La regla general en los sistemas de producción de animales es establecer un núcleo reproductivo con un $N_{\mathrm{e}}$ de 50 o 500 individuos, lo cual contribuiría a disminuir la tasa de endogamia, o mantener el $90 \%$ de la variabilidad genética durante 100 
años, respectivamente (Soulé et al. 1986). El $N_{\mathrm{e}}$ depende del tamaño de la población fundadora, de la tasa de crecimiento poblacional $(\lambda)$ y del tiempo generacional, características peculiares que puede presentar una población en un determinado espacio y tiempo (Rieman \& Allendorf 2001). Por tanto, resulta necesario determinar el $N_{\mathrm{e}}$ específicamente para las poblaciones de cocodrilos en cautiverio.

Uno de los propósitos de este trabajo es presentar una propuesta para el manejo genético de $C$. moreletii que sirva como base para el establecimiento de núcleos reproductivos en las UMAs de reciente creación, incluso en aquellas UMAs donde ya se esté reproduciendo la especie, con la finalidad de lograr ejemplares viables genéticamente para repoblar los hábitats de donde fueron extirpadas sus poblaciones. Por estas razones, los objetivos del presente trabajo son estimar y analizar el tamaño mínimo viable (TMV), el tamaño efectivo de población $\left(N_{e}\right)$, y la tasa de incremento de endogamia por generación $(\Delta \mathrm{F})$, tomando como base una población de $C$. moreletii mantenida en cautiverio en una UMA establecida en el trópico mexicano. Con esta información es posible estimar los tamaños poblacionales a establecerse en las UMAs, de tal manera que se garantice que los progenitores reproductivos extraídos de su hábitat silvestre transmitan las mismas características de variación genética a su progenie, y así asegurar que los ejemplares liberados resistan y se adapten a las condiciones ambientales.

\section{MATERIAL Y MÉTODOS}

El presente trabajo examinó mediante un modelo de simulación los posibles escenarios de extinción y de incremento poblacional a partir del TMV con su respectivo análisis de viabilidad poblacional (AVP); el $N_{e}$ fue estimado con métodos directos y ecológicos; en los casos donde se determinó la $\Delta F$, esta fue calculada en función del tamaño efectivo de población y en función del número de reproductores. Para determinar estos parámetros, se utilizó como referencia la población de $C$. moreletii hacinada para su aprovechamiento comercial en la UMA Cacahuatal (INE/CITES/ DFYFS-CRIN-0069-SIN/99), ubicada en el poblado José Ingenieros, municipio de La Antigua, que se localiza en la región centro del estado de Veracruz, México.

La población en cautiverio de C. moreletii de la UMA Cacahuatal está conformada por cuatro sub-poblaciones que provienen de distintas regiones geográficas de México: Tezonapa y Gutiérrez Zamora, ambas del estado Veracruz, cuyos cocodrilos fueron capturados en vida silvestre, y de Puerto Vallarta, Jalisco y Villa Juárez, Sinaloa, cocodrilos que nacieron en UMAs establecidas en esos municipios y posteriormente enviados a la UMA Cacahuatal; los progenitores de las últimas dos poblaciones son cocodrilos extraídos del rango de distribución natural en México. Lamentablemente se desconoce la localidad o el registro geográfico del sitio donde estos progenitores fueron capturados. La población se mantiene en acuaterrarios semi-naturales con una extensión alrededor de 8 ha, delimitada por malla galvanizada, con lagunetas y sitios con 
vegetación que son ocupados por los ejemplares para realizar las actividades de comportamiento como la reproducción, anidamiento, alimentación y termorregulación.

De la población en cautiverio en la UMA Cacahuatal se obtuvo el tamaño poblacional censal $(N)$, el número de hembras $\left(N_{h}\right)$ y número de machos $\left(N_{m}\right)$, y estos fueron clasificados de acuerdo a su longitud total (LT: medida desde la punta del hocico al final de la cola) en cuatro categorías de talla: crías (LT $<50 \mathrm{~cm}$ ), juveniles (LT $>50$ $\mathrm{y}<90 \mathrm{~cm}$ ), sub-adultos $(\mathrm{LT}>91 \mathrm{y}<130 \mathrm{~cm})$ y adultos $(\mathrm{LT}>131 \mathrm{~cm})$. El sexo se determinó por tacto cloacal (Chabreck 1963), y se consideraron sexualmente maduros (reproductivos) aquellos ejemplares con LT $>130 \mathrm{~cm}$.

El TMV se determinó siguiendo la propuesta de Shaffer (1981) y Harris et al. (1987). Para esto, se generó un modelo de simulación demográfico para la población de $C$. moreletii de la UMA Cacahuatal, con la finalidad de determinar la probabilidad de sobrevivencia en los próximos 50 años con 100 réplicas. En las simulaciones de dicho modelo se consideró como factor la estocasticidad ambiental y el efecto de la densidad poblacional. Para determinar la probabilidad de sobrevivencia se generó un escenario, el cual estuvo estructurado con matrices de proyección poblacional (Caswell 2001), integrando en cada categoría de talla de los cocodrilos sus probabilidades de sobrevivencia $(90 \%)$, fertilidad (tamaño de nidada $=30$ huevos en promedio), abundancia inicial ( $N=1145$ ejemplares), capacidad de carga $(k=5000$ ejemplares) y una $\lambda$ (tasa de crecimiento poblacional) máxima de $1 \pm 0.01$. Estos valores (sobrevivencia, fertilidad, abundancia inicial, capacidad de carga y tasa de crecimiento poblacional) son los que ocurren en la población de $C$. moreletii de la UMA Cacahuatal. En este escenario también se implementó una tasa de cosecha anual de 30 adultos y 25 crías y una tasa de reintroducción de 35 adultos (Traill et al. 2007). Para determinar el riesgo de extinción, se generó otro escenario con las mismas características que el escenario anterior pero solo se diferenció en la aplicación de una $\lambda=1 \pm$ 0.2. Los modelos de simulación fueron corridos en el software RAMAS ${ }^{\circledR}$ EcoLab 2.0 (Akcakaya et al. 1999).

Una vez que se obtuvo el TMV, se aplicó un análisis de viabilidad poblacional (AVP) de los primeros 5 años de persistencia, basado en 10 pruebas utilizando simulación Monte Carlo. En el AVP se utilizó la abundancia inicial considerando el TMV (ver Resultados) con $\lambda=1 \pm 0.01$ y se obtuvo su probabilidad de extinción utilizando números aleatorios aplicados a la desviación típica de $\lambda$.

El tamaño efectivo de población $\left(N_{e}\right)$, fue calculado mediante siete métodos siguiendo la propuesta de Moreno (2007), que incluyen métodos directos (incisos a, b y c) y métodos ecológicos y demográficos (incisos d, e, f y g) para poblaciones aisladas y con generaciones sobrelapadas (ver ecuaciones en el Cuadro 2):

a) Sexos separados (ecuación 1), aplicable para organismos dioicos. Por tanto, el $N_{e}$ depende del número de individuos de cada sexo (Hedrick 2000). 
b) Fluctuaciones anuales en el $N_{e}$ durante 100 años (ecuación 2), generadas mediante números aleatorios (Merrel 1981). En este caso, el $N_{e}$ se verá afectado por las fluctuaciones demográficas, por lo cual se considera la media armónica de los tamaños poblacionales como el $N_{e}$ (Kalinowski \& Waples 2002).

c) Utilizando la tasa de incremento de endogamia (ecuación 3), que está referida al apareamiento no aleatorio, donde la probabilidad de que un individuo presente alelos idénticos por descendencia es mayor que para una población panmíctica (Abarca \& López 2007).

d) Proporción de sexos (ecuación 4), que indica que en una población donde la reproducción es al azar, con un número desigual de hembras y machos, el tamaño de la población actual es el $N_{e}$ (Eding \& Laval 1999).

e) Diferencias en el éxito reproductivo (ecuación 5), que expresa que la progenie por familia no tiene una distribución Poisson (Eguiarte et al. 1993).

f) Estructura de edades (ecuación 6), la cual considera que individuos de distintas edades tendrán una contribución diferencial a las siguientes generaciones (Eguiarte et al. 1993, Hedrick 2000).

g) Estructura de edades y diferencias en fecundidad (ecuación 7), el cual supone una estructura estable de edades y que la población no está creciendo (Eguiarte et al. 1993).

La tasa de incremento de endogamia por generación $(\Delta F)$ fue calculada en función del tamaño censal $(N)$ con la ecuación $\triangle F N=1 / 2 \mathrm{~N}$ (Eding \& Laval 1999), y en función del $N_{e}: \Delta N_{e}=1 / 2 N e$ (Crow \& Kimura 1970).

\section{RESULTADOS}

Se encontró que para las poblaciones en cautiverio de $C$. moreletii debe establecerse un TMV de 200 ejemplares, con lo que se tendría una densidad poblacional proyectada de alrededor de 9000 individuos ( 1.8 cocodrilos $/ \mathrm{m}^{2}$ ), lo que garantiza su supervivencia a lo largo de 50 años (Figura 1), esto sin tomar en cuenta la capacidad de carga que pudiera soportar el espacio en cautiverio. Por otra parte, el AVP mostró que con este TMV se puede asegurar su sobrevivencia en los primeros 5 años, siempre que se mantenga un valor de $\lambda=1 \pm 0.01$, al menos hasta la réplica 10 , pues en esta se observa un descenso poblacional moderado (Figura 2, línea roja con $\lambda=0.9$ ). A pesar de este descenso demográfico, se obtuvo un promedio de $\lambda=1.3$, lo que indica que la población se encuentra demográficamente estable. De hecho, cuando se aplicó un incremento a la desviación típica de $\lambda$ del TMV, la probabilidad de extinción de la población fue mayor. Solo se presentó un riesgo de extinción del $20 \%$ cuando se presentó una desviación típica de $\lambda$ igual a 0.5 y 0.6 , y $100 \%$ de probabilidad de extinción cuando la desviación típica de $\lambda$ fue de 1.9 (Cuadro 1). 


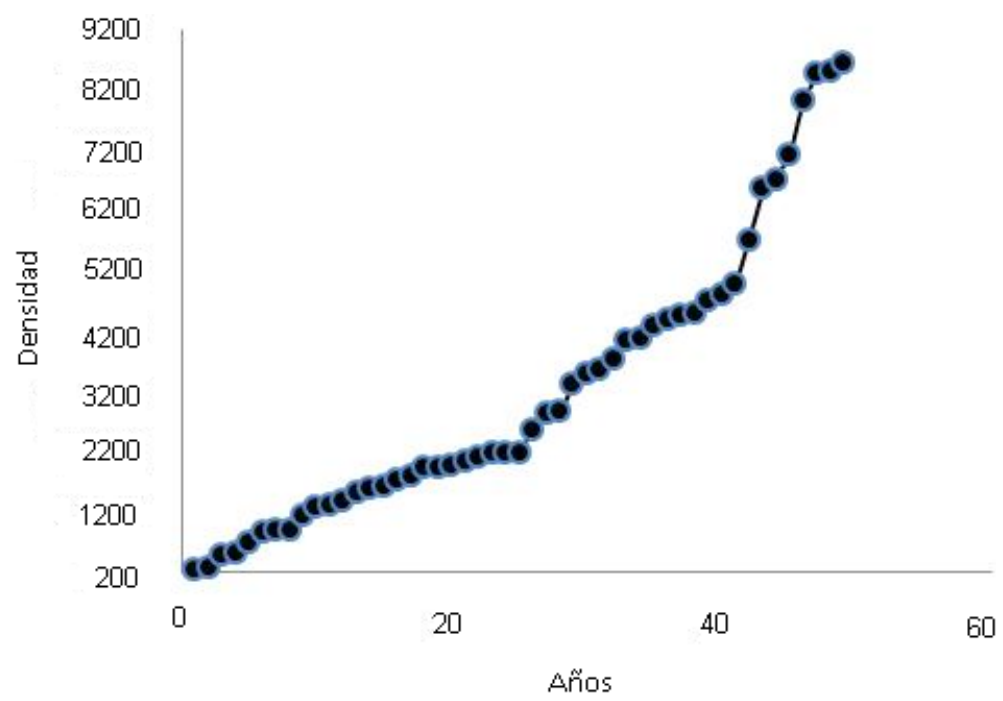

Figura 1. Proyección del incremento en la densidad poblacional a partir del TMV de 200 ejemplares de Crocodylus moreletii durante un periodo de 50 años en la UMA Cacahuatal.

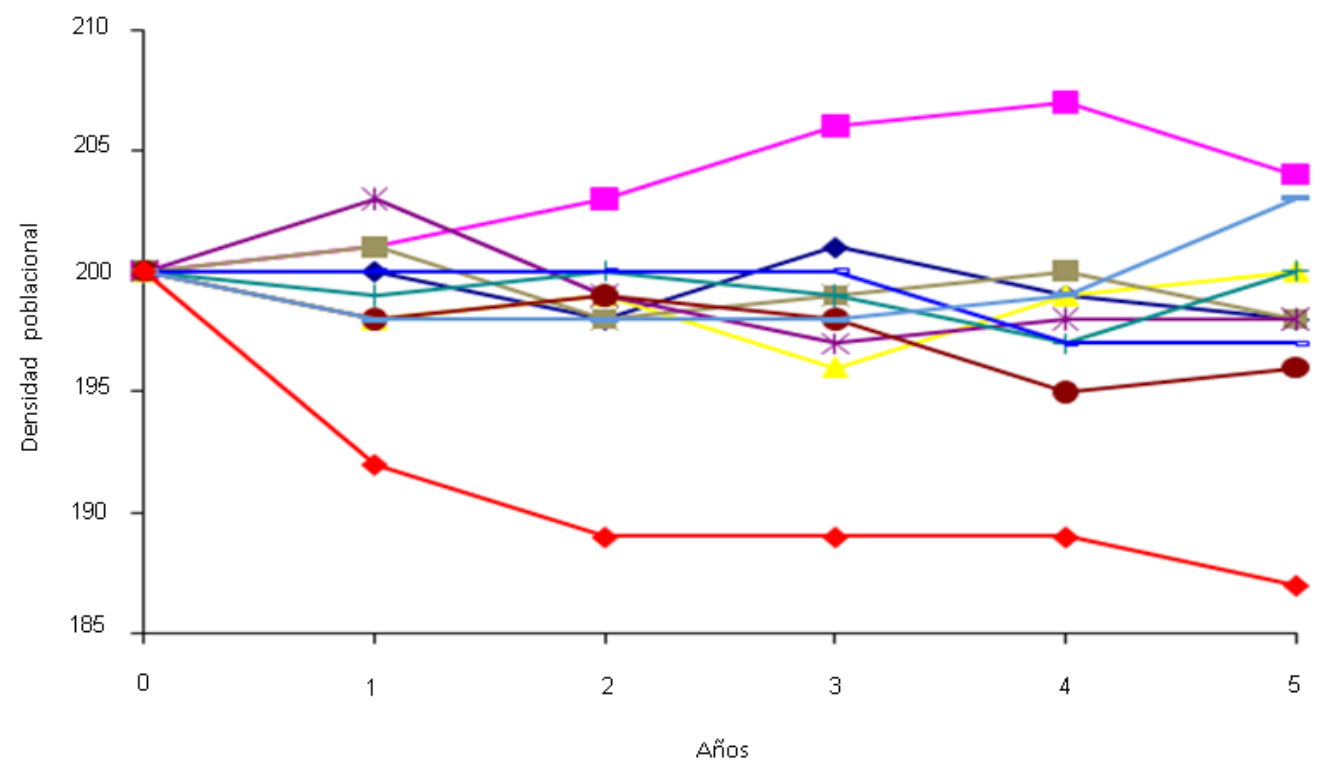

Figura 2. Análisis de viabilidad de la población de Crocodylus moreletii en la UMA Cacahuatal, tomando como base el TMV de 200 individuos y una $\lambda=1 \pm 0.01$. Los símbolos de cada línea corresponden al año (año 1, 2, 3, 4, 5) de prueba en un periodo generacional (10 generaciones). 
Cuadro 1. Análisis de viabilidad poblacional del tamaño mínimo viable utilizando simulación Monte Carlo aplicada a la desviación típica de $\lambda$ de una población de Crocodylus moreletii en cautiverio.

\begin{tabular}{cccc}
\hline N(0) & Media $\lambda$ & Desviación típica de $\boldsymbol{\lambda}$ & $\begin{array}{c}\text { Probabilidad de } \\
\text { extinción }\end{array}$ \\
\hline 200 & 1.0 & 0.2 & 0.0 \\
200 & 1.0 & 0.3 & 0.0 \\
200 & 1.0 & 0.4 & 0.0 \\
200 & 1.0 & 0.5 & 0.2 \\
200 & 1.0 & 0.6 & 0.2 \\
200 & 1.0 & 0.7 & 0.5 \\
200 & 1.0 & 0.8 & 0.5 \\
200 & 1.0 & 0.9 & 0.6 \\
200 & 1.0 & 1.0 & 0.6 \\
200 & 1.0 & 1.3 & 0.8 \\
200 & 1.0 & 1.9 & 1.0 \\
\hline
\end{tabular}

El primer escenario del modelo de simulación manifestó que con el tamaño poblacional de C. moreletii en la UMA Cacahuatal se presentará una estabilidad de la densidad poblacional a través de los 50 años simulados (Figura 3). En este caso, los 1145 cocodrilos que se mantienen en cautiverio, tienen un $95 \%$ de probabilidad de sobrevivir (Figura 4). En lo que respecta al tamaño poblacional de C. moreletii que se mantiene en la UMA Cacahuatal, la probabilidad de extinción disminuye conforme aumenta la población (Figura 5). El escenario dos evidenció que la población de $C$. moreletii en la UMA Cacahuatal presenta mayores probabilidades de tendencia a la extinción (cuando se aplica un mayor incremento a la varianza de $\lambda$ ). En este sentido, la densidad se encuentra por encima de la capacidad de carga que puede soportar el área (Figura 6); por tanto, cada umbral poblacional disminuye (Figura 7), y el riesgo de extinción se incrementa (Figura 8).

En el Cuadro 2 se presenta el tamaño efectivo de población $\left(N_{e}\right)$ estimado con métodos directos y ecológico-demográficos. La ecuación 1 determinó que el $N_{e}$ se reduce a 400 ejemplares cuando existe una proporción igual en el número de machos y hembras. Al respecto, si la población presenta fluctuaciones mínimas en los parámetros demográficos por un periodo de 100 años presentará un $N_{e}$ de 927 cocodrilos (ecuación 2, Cuadro 2). Por otra parte, cuando se incrementa la $\Delta F$ se reduce el $N_{e}$ (ecuación 3, Cuadro 2). Con una proporción desigual de sexos el $N_{e}$ tiende a reducirse (ecuación 4, Cuadro 2), y suponiendo que durante el cautiverio la reproducción solo la efectúan algunos machos dominantes o un pequeño grupo de reproductores también se presenta una reducción en el $N_{e}$ (ecuación 5, Cuadro 2); asimismo, suce- 


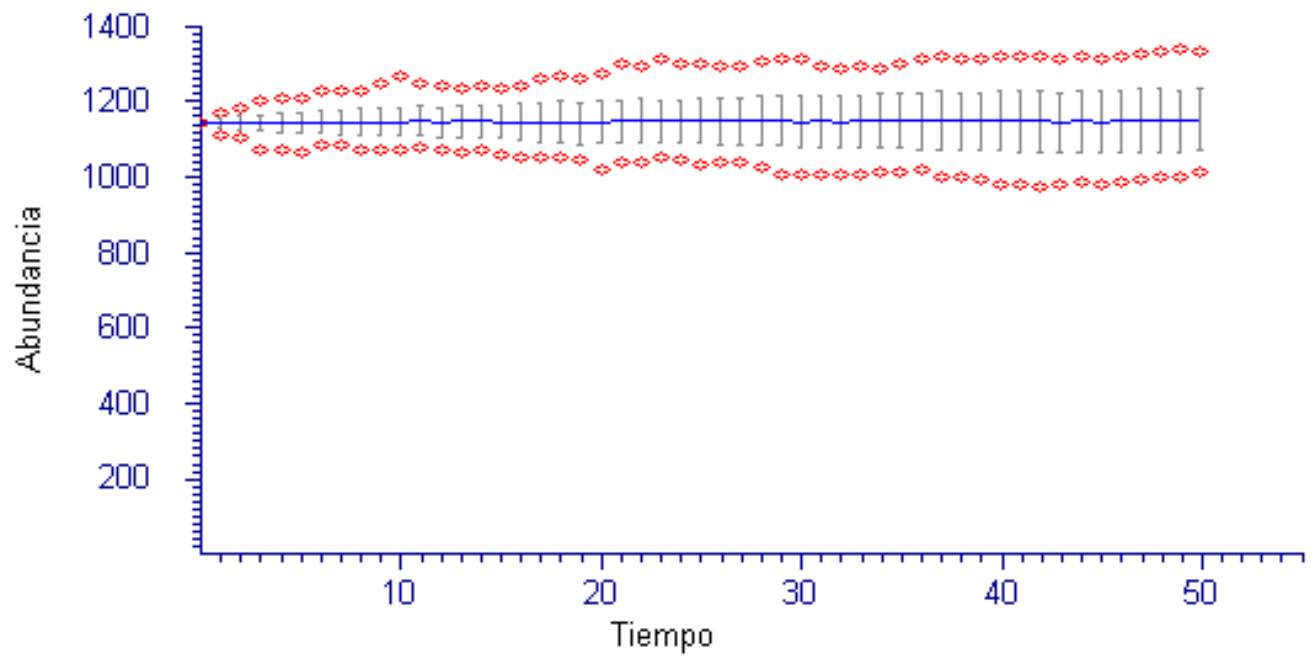

Figura 3. Viabilidad de la población de Crocodylus moreletii de la UMA Cacahuatal durante un periodo de 50 años. Los romboides (en rojo), y las barras verticales denotan el valor del rango esperado y observado, respectivamente; la línea de tendencia expresa el promedio de la abundancia poblacional.

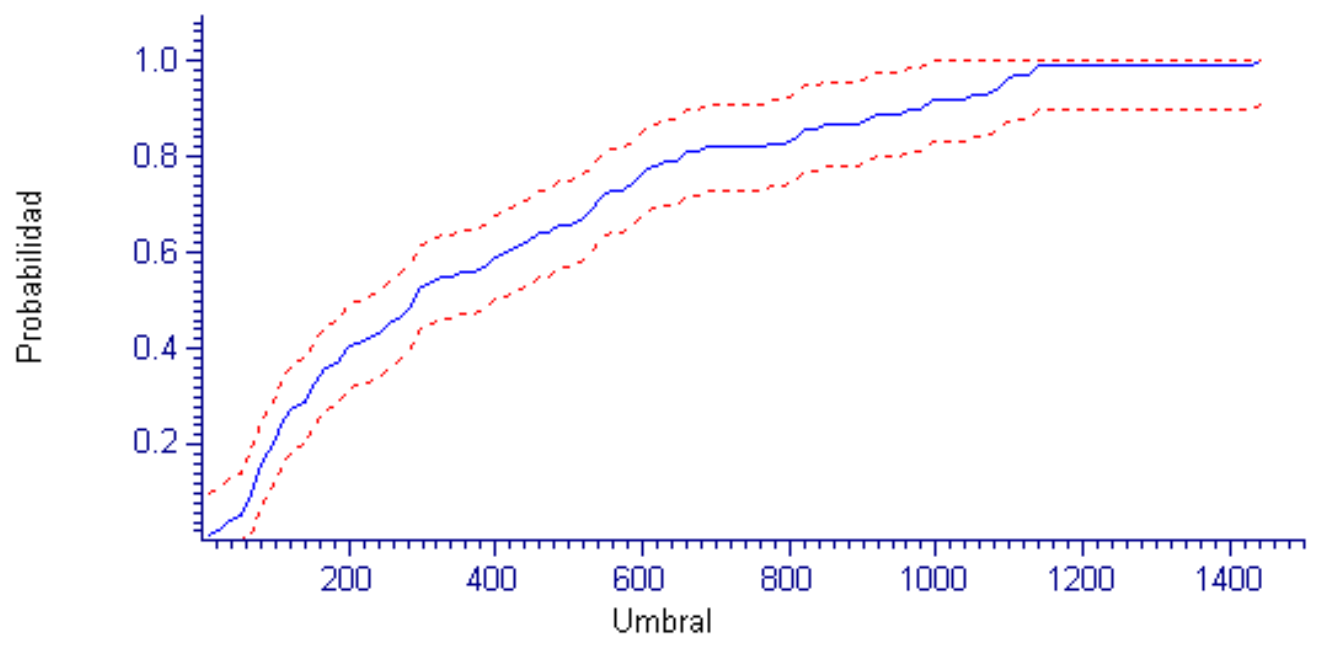

Figura 4. Probabilidad de incremento de la población de Crocodylus moreletii en la UMA Cacahuatal. El rango del umbral poblacional es de 60 y 1400 cocodrilos, que representan una probabilidad de incremento que va de 0.2 a 0.99 , respectivamente. La línea punteada roja denota el valor máximo y mínimo (por encima y debajo de la línea de tendencia, respectivamente) del umbral esperado de la población. 


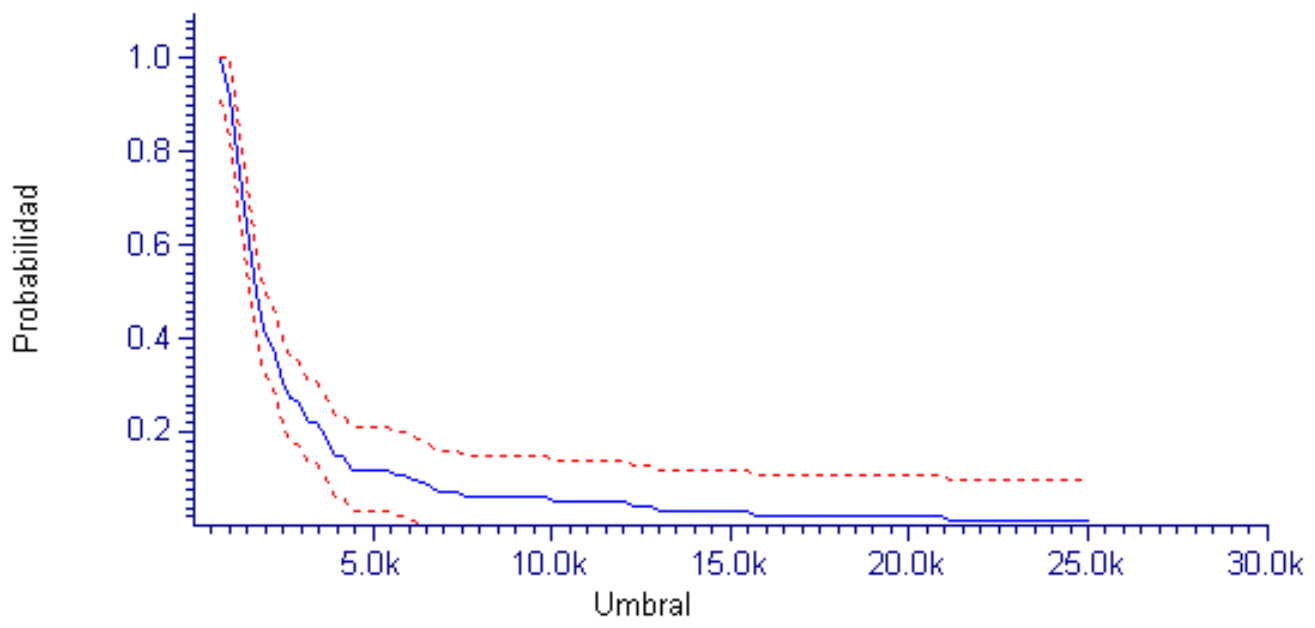

Figura 5. Disminución en el riesgo de extinción de la población de Crocodyulus moreletii en cautiverio en la UMA Cacahuatal. El valor del rango del umbral de la capacidad de carga $(k)$ es de $2.0 k$ y $25 k$ que representa una probabilidad de extinción de la población entre 0.9 y 0 , respectivamente. La línea punteada roja denota el valor máximo y mínimo (por encima y debajo de la línea de tendencia, respectivamente) del umbral esperado de $k$.

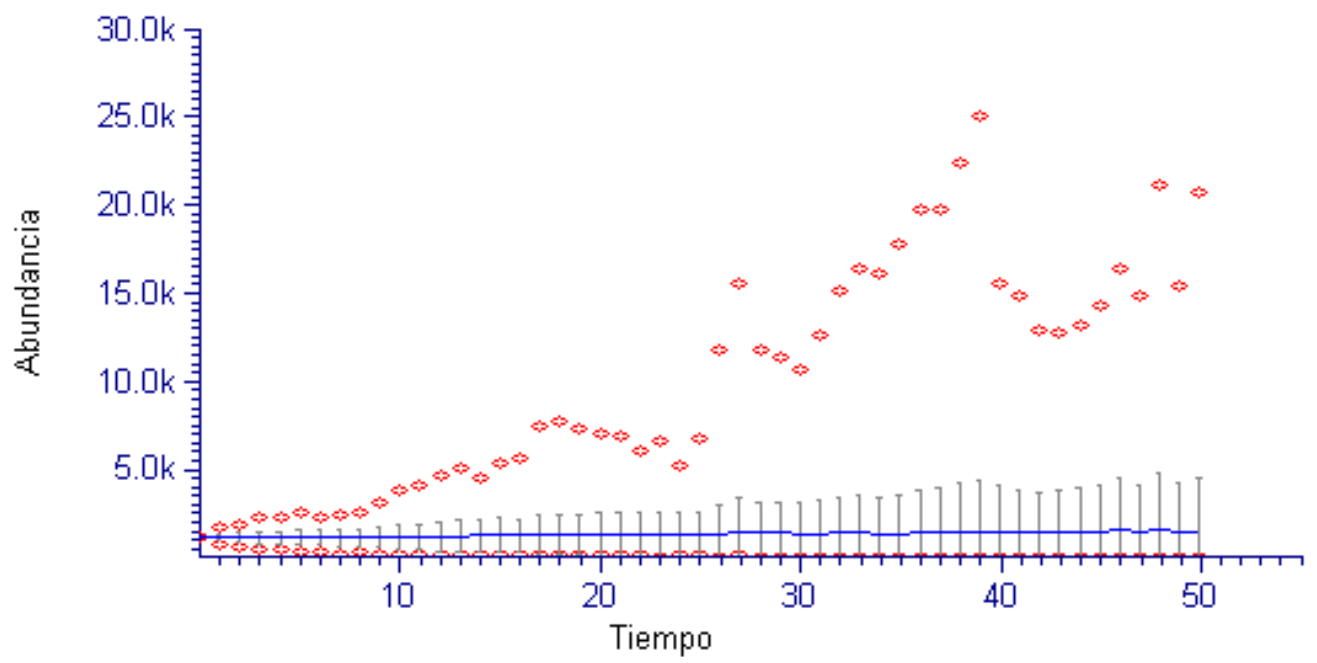

Figura 6. Disminución en la abundancia de la población de Crocodyulus moreletii en la UMA Cacahuatal por sobrepasar la capacidad de carga $(k)$. Los romboides (en rojo) indican los valores del rango esperado de $k$ a través del tiempo; las barras verticales denotan los valores del rango observado de $k$, y la línea de tendencia expresa el promedio de $k$. 


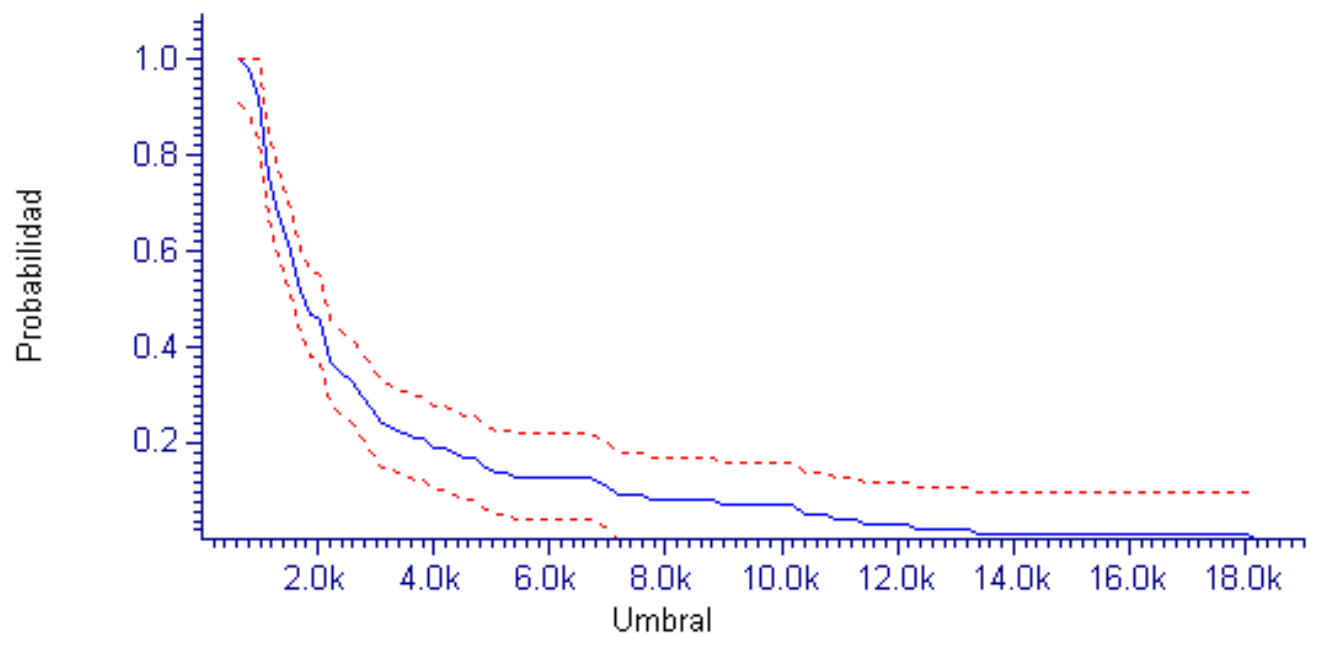

Figura 7. Disminución en la probabilidad del crecimiento poblacional de Crocodylus moreletii en la UMA Cacahuatal. Los valores del rango del umbral de la capacidad de carga $(k)$ son de $1.2 k$ y $18 k$, que representan una probabilidad de disminución poblacional que va de 0.9 a 0 , respectivamente. La línea punteada roja denota el valor máximo y mínimo (por encima y debajo de la línea de tendencia, respectivamente) del umbral esperado de $k$.

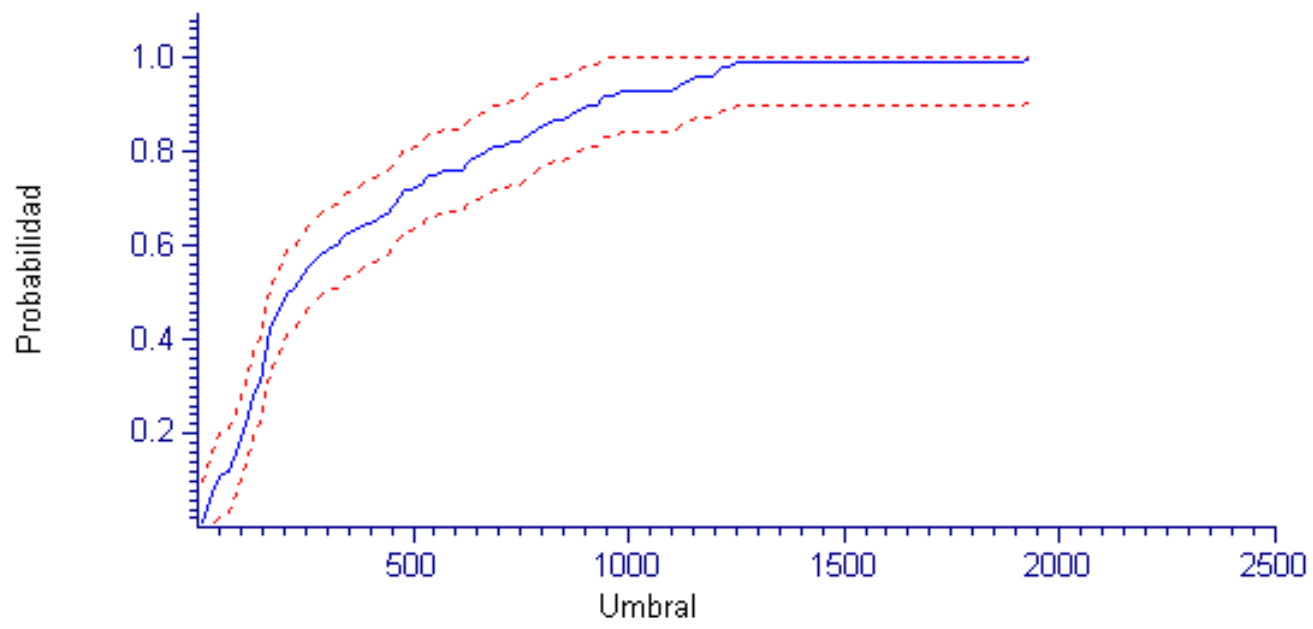

Figura 8. Aumento en el riesgo de extinción en la población de Crocodylus moreletii de la UMA Cacahuatal. El valor del umbral poblacional inicial es de $\sim 40$ cocodrilos que conllevaría a la extinción de la población. La línea punteada roja denota el valor máximo y mínimo (por encima y debajo de la línea de tendencia, respectivamente) del umbral esperado de la población. 
Serna-Lagunes \& Díaz-Rivera: Variación genética de Crocodylus moreletii

Cuadro 2. Métodos para estimar el tamaño efectivo de población $\left(N_{e}\right)$ para Crocodylus moreletii en cautiverio.

\begin{tabular}{ccccccccc}
\hline & Ecuación & $N_{m}$ & $N_{h}$ & $N_{m}: N_{h}$ & $N$ & $N_{e}$ & $\begin{array}{c}\Delta F_{N} \\
(\%)\end{array}$ & $\begin{array}{c}\Delta F_{N e} \\
(\%)\end{array}$ \\
\hline 1 & $1 / N_{e}=1 / 4 N_{h}+1 / 4 N_{\mathrm{m}} \mathrm{o} N_{e}=$ & 572 & 572 & $1: 1$ & 1144 & 400 & 5.7 & 5.7 \\
& $4 N_{h} N_{\mathrm{m}} / N_{h}+N_{m}$ & & & & & & & \\
2 & $1 / N_{e}=1 / T\left(1 / N_{1}+1 / N_{2}+1 / N_{3}+\ldots 1 / N_{\mathrm{T}}\right)$ & $310^{\dagger}$ & $789^{\dagger}$ & $1: 2.5$ & $1099^{\dagger}$ & $927^{*}$ & 5.4 & 4.6 \\
3 & $N_{e}=N /(1+\Delta F)$ & 335 & 809 & $1: 2.4$ & 1144 & 171 & 5.7 & 5.4 \\
4 & $1 / N_{e}=1 / 4(1 / \mathrm{Nm}+1 / \mathrm{Nh})$ & 335 & 810 & $1: 2.4$ & 1145 & 349 & 5.7 & 4.7 \\
5 & $N_{e}=4\left(N_{0}-2\right) \mathrm{L} / V+2$ & - & - & - & - & 392 & - & 1.9 \\
6 & $N_{e}=N_{r} \mathrm{~L}$ & - & - & - & - & 275 & - & 1.3 \\
7 & $N_{e}=\mathrm{N}_{0} \mathrm{~L}_{\mathrm{i}}$ & - & - & - & - & 146 & - & 0.7 \\
\hline
\end{tabular}

$N_{m}$ y $N_{h}=$ número machos y hembras, respectivamente; $N_{m}: N_{h}=$ relación sexual; $N=$ tamaño censal; $N_{e}=$ tamaño efectivo de población; $\Delta F_{N} \mathrm{y} \Delta F_{N e}$ = tasa de incremento de endogamia por generación en función del $N$ y $N_{e}$, respectivamente. "Promedio de individuos; "Media armónica; $T=$ número de generaciones (100); $\Delta F=$ tasa de incremento de endogamia; $N_{0}=$ Número de individuos nacidos en una temporada (30); $\mathrm{L}=$ tiempo generacional (11 años); $V$ = varianza en el éxito reproductivo (1.5 individuos); $N_{r}=$ número de individuos nacidos en un año dado que llegan a reproducirse (25); i = suma de todas las edades del cuadrado de la sobrevivencia específica de cada edad por la fecundidad específica para las edades (441).

de algo similar si se cuenta con cocodrilos con distintas clases de tamaño (ecuación 6, Cuadro 2), pero se presenta una reducción aun mayor si durante el cautiverio se tienen cocodrilos con diferentes clases de tamaño y solo algunos de estos se pueden reproducir (ecuación 7 , Cuadro 2).

\section{DISCUSIÓN}

La viabilidad de poblaciones está influida por fuerzas conductoras como la densodependencia y la variabilidad ambiental y se puede considerar que una población no se encuentra amenazada por riesgo de extinción cuando esta presenta valores por encima del 95\% de probabilidad de sobrevivir durante los 50 años posteriores (Zabel et al. 2006). En este sentido, nuestras estimaciones indican que si se establece un tamaño mínimo de población de 200 cocodrilos, la probabilidad de sobrevivir durante los próximos 50 años es alta. Con este tamaño poblacional, se presentó un incremento exponencial de $\lambda$. Esto indica que se puede establecer una tasa de cosecha para comercializar los cocodrilos que no pueda soportar el área. Otra opción para utilizar estos cocodrilos, es la selección de ejemplares para establecer otros núcleos reproductivos con el mismo TMV.

Cuando comparamos el tamaño de población mínimo viable entre poblaciones de cocodrilianos encontramos valores contrastantes. Por ejemplo, la población silvestre de Crocodylus intermedius de Venezuela (Seijas \& Chavez 2000) y la población de 
Crocodylus rhombifer en Cuba (Ramos et a., 2010), presentan un TMV de $547 \mathrm{y}$ 3000 cocodrilos, tamaños superiores al estimado en este estudio. Estas diferencias se atribuyen a que $C$. intermedius y $C$. rhombifer tienen una mayor superficie de distribución, de $195.6 \mathrm{~km}^{2}$ y $360 \mathrm{~km}^{2}$ respectivamente, en comparación con el área en la que se mantiene hacinada la población de C. moreletii. Esta comparación enfatiza que el TMV calculado para $C$. moreletii está relacionado con el área destinada a la explotación, y por tanto, es necesario utilizar esta variable para asegurar la viabilidad de la población y el proyecto de explotación.

Dado que la población de $C$. moreletii se mantiene en cautiverio, generalmente es fácil controlar la denso-dependencia y la variabilidad ambiental que influye directamente en la fluctuación anual del $N_{e}$. Sin embargo, esto fue parcialmente cierto, debido a que se presentó una reducción del $16 \%$ del $N_{e}$ calculado mediante la ecuación 2 , que fue con la que se obtuvo un mayor $N_{e}$. Pero aún a pesar de esta reducción, se mantiene un número considerable de cocodrilos efectivos para reproducirse, en comparación con las otras estimaciones, las cuales presentan mayores reducciones en el $N_{e}$. Debido a que en las otras estimaciones se incluye una proporción de sexos similar (ecuación 1), y sesgada (ecuaciones 3 y 4) por un mayor número de hembras, también se reduce el $N_{e}$ (Stiver et al. 2008). De hecho, por presentar varianza en el éxito reproductivo en cada periodo generacional, también hay una reducción similar en el tamaño efectivo (Hedrick 2005). Esto puede deberse a que los parámetros poblaciones no se encuentran en equilibrio y merman el tamaño efectivo (Reed et al. 2003).

Si en la UMA Cacahuatal se conservan los parámetros poblacionales a través de las fluctuaciones anuales con los que se obtiene un $N_{e}$ de 927 cocodrilos, se estarían cumpliendo los supuestos propuestos por Franklin (1980), quien indica que necesariamente se requiere tener un $N_{e} \sim 500$ individuos para mantener los niveles de variación genética. En poblaciones de C. niloticus con valores de $N_{e} \sim 480$, se observó la pérdida de una proporción importante de su variación genética (Bourquin 2007).

La principal preocupación de los conservacionistas es que poblaciones pequeñas o mantenidas en cautiverio constantemente sufren la pérdida de la variación genética causada por los efectos de la endogamia (Lande 1988). Además, en poblaciones en cautiverio la endogamia generalmente es inevitable debido al reducido número de reproductores efectivos (Pray et al. 1994), siendo un factor que incrementa la tasa de pérdida de heterocigosis (Wright 1931). Nuestras estimaciones indican que el $N_{e}$ de la población de C. moreletii en la UMA Cacahuatal es suficiente para garantizar la estabilidad de la variación genética. Esto es consistente cuando se corrobora con los valores de la tasa de incremento de endogamia $(\Delta F)$, la cual obtuvo valores menores al 10\% (ver Cuadro 2). En este contexto, Soulé (1980) y Soulé et al. (1986), sugieren que se debe preservar por lo menos el 90\% de variación genética durante 200 años. En este sentido, a una $\Delta F$ menor al $10 \%$, se disminuye la pérdida de heterocigosis, y consecuentemente disminuye la velocidad con que se fijan los genes recesivos dele- 
téreos (Futuyma 1986), generando cocodrilos genéticamente viables para su reintroducción. Es decir, con el $N_{e}$ que se estimó para $C$. moreletii, se puede considerar que la progenie no presentaría una reducción de su variación genética. Por tanto, las crías tendrían la misma, o una similar proporción de variación genética que los progenitores extraídos del hábitat silvestre, y esto apoyaría en cierta medida, la liberación de crías al hábitat natural con el reservorio genético que permitirá su adaptación.

En conclusión, si se quiere implementar un programa de gestión a través de una UMA para la explotación de $C$. moreletii, se puede iniciar con el establecimiento de 200 cocodrilos con lo que se aseguraría la viabilidad de la población en los futuros 50 años. Si la finalidad es la conservación de la especie y aunado a esto, preservar la variabilidad genética, es necesario establecer un núcleo reproductivo con $N_{e}$ mayor de 900 cocodrilos y con una proporción de sexos de 1 macho por 2.5 hembras, con los cuales se minimizarían los efectos del incremento de endogamia y la pérdida de la heterocigosidad, a través de reducidas $\Delta F$. Estos parámetros demográficos preservarán el reservorio genético de la especie mediante la herencia de los mismos niveles de variabilidad genética a su descendencia. Se sugiere que antes de llevar a cabo un programa de repoblación en los hábitats donde fue extirpada la especie, se analice una muestra representativa de los progenitores y de los descendientes por liberar usando marcadores moleculares como los microsatélites, con el propósito de tener una estimación más confiable y valorar la variación genética de los cocodrilos a liberar.

AGRADECIMIENTOS. A los representantes de la UMA Cacahuatal por las facilidades brindadas para realizar el presente estudio. A la Secretaría de Medio Ambiente y Recursos Naturales de México por otorgar la licencia especial de colecta (SGPA/DGVS/07875) para realizar la captura de los cocodrilos. Los autores agradecemos las sugerencias de los dos árbitros anónimos quienes contribuyeron sustancialmente para mejorar la estructura y la presentación de la información de esta investigación.

\section{LITERATURA CITADA}

Álvarez del Toro, M. \& L. Sigler. 2001. Los Crocodylia de México. Instituto Mexicano de Recursos Naturales Renovables y Procuraduría Federal de Protección al Ambiente. México, D.F.

Abarca, G. C. A. \& V. A. López. 2007. La estimación de la endogamia y la relación entre la tasa de fecundación cruzada y los sistemas reproductivos en plantas con flores: una interpretación de su significado evolutivo, pp. 183-214. In: L. Eguiarte, V. Souza \& X. Aguirre (Eds.). Ecología Molecular. Instituto Nacional de Ecología. México, D. F.

Akcakaya, H. R., M. A. Burgman \& L. R. Ginzburg. 1999. Applied population ecology: principles and computer exercises using RAMAS EcoLab 2.0. Sinauer Associates, Inc., Sunderland, Massachusetts.

Caswell, H. 2001. Matrix populations models: construction, analysis and interpretation. Sinauer Associates, Inc., Sunderland, Massachusetts.

Bourquin, S. L. 2007. The population ecology of the Nile Crocodile (Crocodylus niloticus) in the Panhandle Region of the Okavango Delta, Botswana. Ph.D. Thesis. Stellenbosch University, South Africa. 
Caballero, A. 1994. Development in the prediction of effective population size. Heredity, 73: 657679.

Chabreck, R. 1963. Methods of capturing, marking and sexing alligators. Proceedings of the Annual Conference Southeastern Association of Game and Fish Commission, 17: 47-50.

Crow, J. F. \& M. Kimura. 1970. An introduction to population genetics theory. Harper \& Row, New York.

Domínguez-Laso, J. 2006. Determinación del estado actual de las poblaciones silvestres del cocodrilo de pantano (Crocodylus moreletii) en México y evaluación de su estatus en la CITES. Instituto de Historia Natural y Ecología, Sistema Nacional de Información y Comisión Nacional para el Conocimiento y Uso de la Biodiversidad. México, D.F.

Eding, J. H. \& G. Laval. 1999. Measuring genetic uniqueness in livestock. Pp. 33-58. In: J.K. Oldenbroek (ed). Genebanks and the conservation of farm animal genetic resources. ID-DLO Institute for Animal Science Health. A. B. Lelystad, Netherlands.

Eguiarte, L. E., A. Búrquez, J. Rodríguez, M. Martínez-Ramos, J. Sarukhán \& D. Piñero. 1993. Direct and indirect estimates of neighborhood and effective population size in a tropical palm, Astrocaryum mexicanum. Evolution, 47: 75-87.

Ferrer, M., I. Newton \& M. Pandolfi. 2009. Small population and offspring sex-ratio deviations in Egles. Conservation Biology, 23: 1017-1025.

FitzSimmons, N., J. C. Buchan, P. V. Lam, G. Polet, T. T. Hung, N. Q. Thang \& J. Gratten. 2002. Identification of purebred Crocodylus siamensis for reintroduction in Vietnam. Journal of Experimental Zoology, 294: 373-381.

Forstner, M. \& J. M. Forstner. 2002. Aplicaciones del DNA en la conservación de los cocodrilianos. Pp. 99-117. In: L. M. Verdade \& A. Larriera (Eds.). La conservación y el manejo de caimanes y cocodrilos de América Latina. C.N. Editorial, Piracicaba, Brasil.

Futuyma, D. J. 1986. Evolutionary biology. Sinauer Associates, Inc., Sunderland, Massachusetts.

Flint, N. S., F. H. Van der Bank \& J. P. Grobler. 2000. A lack of genetic variation in commercially bred Nile crocodile (Crocodylus niloticus) in the North-West Province of South Africa. Water SA, 26: $105-110$.

Frankham, R. 1995. Effective population size/adult population size ratios in wildlife: a review. Genetical Research, 66: 95-107.

Frankham, R. 1996. Relationship of genetic variation to population size in wildlife. Conservation Biology, 10: 1500-1508.

Frankham, R. 2002. Genetics and extinction. Biological Conservation, 126: 131-140.

Frankham R., J. D. Ballou \& D. A. Briscoe. 2002. Introduction to conservation genetics. Cambridge, Reino Unido.

Franklin, I. R. 1980. Evolutionary change in small populations. Pp. 135-150. In: M.E. Soulé \& B.A. Wilcox (eds.). Conservation Biology. Sinauer Associates, Inc., Sunderland, Massachusetts.

Glenn, T. C. 2002. DNA tools and resources for crocodilian research. Pp. 211. In: Proceedings of the 16th Working Meeting of the Crocodile Specialist Group of the Species Survival Commission of IUCN \& World Conservation Union (Eds.). Crocodiles. Gland, Switzerland.

Harris, R. B., A. M. Lynn \& M. L. Shaffer. 1987. Sample sizes for minimum viable populations estimation. Conservation Biology, 1: 72-76.

Hartl, D. L. \& A. G. Clark. 1997. Principles of population genetics. Sinauer Associates, Inc., Sunderland, Massachusetts.

Hedrick, P.W. 1995. Gene flow and genetic restoration: the Florida panther as a case study. Conservation Biology, 9: 996-1007.

Hedrick, P. W. 2000. Genetics of populations. Jones \& Bartlett Publishers, Boston. 
Hedrick, P. W. 2005. "Genetic restoration": a more comprehensive perspective than "genetic rescue". Trends in Ecology and Evolution, 20: 109.

Hedrick, P. W. \& S. T. Kalinowski. 2000. Inbreeding depression in conservation biology. Annual Review of Ecology and Systematics, 31: 139-162.

Hoelzel, A. R., J. Halley, S. J. O'Brien, C. Campagna, T. Arnborm, B. Le Boeuf, K. Ralls \& G. A. Dover. 1993. Elephant seal genetic variation and the use of simulation models to investigate historical population bottlenecks. Heredity, 84: 443.

Huchzermeyer, F. W. 2003. Crocodiles: Biology, Husbandry and Diseases. CABI Publishing, Wallingford, Oxfordshire, Reino Unido.

INE. 1997. Programa de conservación de la vida silvestre y diversificación productiva en el sector rural 1997-2000. Instituto Nacional de Ecología y Secretaria de Medio Ambiente y Recursos Naturales. México, D. F.

IUCN, 2010. IUCN Red List of Threatened Species. Version 2010.4. <www.iucnredlist.org>. Consultado 27 de abril 2010.

Kalinowski, T. S. \& R. S. Walpes. 2002. Relationships of effective to census size in fluctuating populations. Conservation Biology, 16: 129-136.

Lande, R. 1988. Genetics and demography in biological conservation. Science, 241: 1455-1460.

Marmontel, M., S. R. Humphrey \& T. J. O'Shea. 2002. Population viability analysis of the Florida Manatee (Trichechus manatus latirostris), 1976-1991. Conservation Biology, 11: 467-481.

Merrel, D. J. 1981. Ecological genetics. Longman, Londres.

Moreno, L. A. 2007. Tamaño efectivo de la población. Pp. 63-86. In: L. Eguiarte, V. Souza \& X. Aguire (eds.). Ecología Molecular. Instituto Nacional de Ecología. México, D. F.

NOM-059-SEMARNAT. 2010. Protección Ambiental -especies nativas de México de flora y fauna silvestres- Categorías de riesgo y especificaciones para su inclusión, exclusión o cambio- Lista de especies en riesgo. Diario Oficial de la Federación, publicado el 30/12/2010. Distrito Federal, México. 77p.

Nunney, L. \& D. R. Elam. 1994. Estimating the Effective Population Size of Conserved Populations. Conservation Biology, 8: 175-184.

Oldenbroek, J. K. 1999. Genebanks and the management of farm animal genetic resources. ID-DLO Institute for Animal Science and Health. A.B. Lelystad, Netherlands.

Payne, R. B. 1990. Natal dispersal, area effects, and effective population size. Journal of Field Ornitology, 61: 396-403.

Pray, L. A., J. M. Schwartz, C. J. Goodnight \& L. Stevens. 1994. Environmental dependency of inbreeding depression: implications for conservation biology. Conservation Biology, 8: 562-568.

Ramos T. R., R. R. Soberón, M. A. Tabet \& J. B. Thorbjarnarson. 2010. Cuban Crocodile Crocodylus rhombifer. Pp. 114-118. In: S.C. Manolis \& C. Stevenson (Eds.) Crocodiles. Status Survey and Conservation Action Plan. Crocodile Specialist Group: Darwin.

Raybould, A. F., R. T. Clarke, J. M. Bond, R. E. Welters \& C. J. Gliddon. 2002. Inferring patterns of dispersal from allele frequency data. Pp. 89-108. In: J.M. Bullok, R. Kenward \& R. Haills (Eds.). Dispersal ecology. Blackwell Science, Oxford, Reino Unido.

Razmaité, V. \& R. Sveistiené. 2003. Minimal and effective population size of conserved Lithuanian farm animals. Ekologija, 1: 34-37.

Reed, H. D., J. J. O'Grady, B. W. Brook, J. D. Ballou \& R. Frankham. 2003. Estimates of minimum viable populations size for vertebrates and factors influencing those estimates. Biological Conservation, 113: 23-34.

Rieman, B. E. \& F. W. Allendorf. 2001. Effective population size and genetic conservation criteria for bull trout. North American Journal of Fisheries Management, 121: 756-764. 
Ross, J. P. 1998. Crocodiles. Status Survey and Conservation Action Plan. 2nd Edition. IUCN/SSC Crocodile Specialist Group. IUCN. Gland, Switzerland and Cambridge, Reino Unido.

Ryman, N. \& L. Laikre. 1991. Effects of supportive breeding on the genetically effective population size. Conservation Biology, 5: 325-329.

Rocha, M. \& J. Gasca. 2007. Ecología molecular de la conservación. Pp. 251-278. In: L. Eguiarte, V. Souza \& X. Aguirre (eds.). Ecología Molecular. Instituto Nacional de Ecología. México, D. F.

SEMARNAT. 1999. Proyecto para la conservación, manejo y aprovechamiento sustentable de los Crocodylia de México (COMACROM). INE-SEMARNAT. México.

Seijas, E. A. \& C. Chávez. 2000. Population status of the Orinoco crocodile (Crocodylus intermedius) in the Cojedes river system, Venezuela. Biological Conservation, 94: 353-361.

Soulé, M. E. 1980. Thresholds for survival: maintaining fitness and evolutionary potential. Pp. 151170. In: M.E. Soulé \& B.A. Wilcox (eds.). Conservation: an evolutionary-ecological perspective. Sinauer Associates, Inc., Sunderland, Massachusetts.

Soulé, M. E., M. Gilpin, W. Conway \& T. Foose. 1986. The millenium ark: how long a voyage, how many staterooms, how many passengers? Zoological Biology, 5: 101-113.

Schultz, C. B. \& P. C. Hammond. 2003. Using population viability analysis to develop recovery criteria for endangered insects: Case study of the Fender's Blue Butterfly. Conservation Biology, 17 : 1372-1385.

Stiver, J. R., A. D. Apa, T. E. Remington \& R. M. Gibson. 2008. Polygyny and female breeding failure reduce effective population size in the lekking Gunnison sage-grouse. Biological Conservation, 141: 472-481.

Shaffer, M. L. 1981. Minimum population size for species conservation. BioScience, 31: 131-134.

Traill, L. W., C. J. A. Bradshaw \& B. W. Brook. 2007. Minimum viable population size: A metaanalysis of 30 years of published estimates. Biological Conservation, 139: 159-166.

Wang, Y. Q., Zhu, W. Q., Huang, L., Zhou, K. Y. \& Wang, R. P. 2006. Genetic diversity of Chinese alligator (Alligator sinensis) revealed by AFLP analysis: An implication on the management of captive conservation. Biodiversity and Conservation, 15: 2945-2955.

Wu, X., Y. Wang, K. Zhou, W. Zhu, J. Nie, C. Wang \& W. Xie. 2002. Genetic variation in captive population of Chinese alligator, Alligator sinensis, revealed by Random Amplified Polymorphic DNA (RAPD). Biological Conservation, 106: 435-441.

Wright, S. 1931. Evolution in mendelian populations. Genetics, 16: 97-158.

Zabel, R. W., M. D. Scheuerell, M. M. McClure \& J. G. Williams. 2006. The interplay between climate variability and density dependence in the population viability of Chinook Salmon. Conservation Biology, 20: 190-200. 